\title{
Generalization of the non-commuting graph of a group via a normal subgroup
}

\author{
Fereshteh Kakeri $^{\mathrm{a}}$, Ahmad Erfanian ${ }^{\mathrm{b}, *}$, Farzaneh Mansoori ${ }^{\mathrm{a}}$ \\ ${ }^{a}$ Ferdowsi University of Mashhad, International Campus, Mashhad, Iran \\ b Department of Pure Mathematics and Centre of Excellence in Analysis on Algebraic Structures, \\ Ferdowsi University of Mashhad, Mashhad, Iran
}

*Corresponding author, e-mail: erfanian@math.um.ac.ir

Received 18 Feb 2015

Accepted 23 May 2016

\begin{abstract}
Let $G$ be a finite group and $N$ be a normal subgroup of $G$. We define an undirected simple graph $\Gamma_{N, G}$ to be a graph whose vertex set is all elements in $G \backslash Z^{N}(G)$ and two vertices $x$ and $y$ are adjacent iff $[x, y] \notin N$, where $Z^{N}(G)=\{g \in G:[x, g] \in N$ for all $x \in G\}$. If $N=1$, then we obtain the known non-commuting graph of $G$. We give some basic results about connectivity, regularity, planarity, 1-planarity and some numerical invariants of the graph which are mostly improvements of the results given for non-commuting graphs. Also, a probability related to this graph is defined and a formula for the number of edges of the graph in terms of this probability is given.
\end{abstract}

KEYWORDS: commutativity degree, graph diameter, girth, strongly regular, planar graph

MSC2010: 05C25 20P05

\section{INTRODUCTION}

There are many graphs which are associated with groups: for instance, prime graphs ${ }^{1}$, non-cyclic graphs $^{2}$, and conjugate graphs ${ }^{3}$. An important one is the non-commuting graph. Let $G$ be a group. Then the non-commuting graph of $G$, denoted by $\Gamma_{G}$, is a graph whose vertices are elements of $G \backslash Z(G)$ and two distinct vertices $x$ and $y$ are adjacent if $x y \neq y x$. Neumann ${ }^{4}$ obtained the first remarkable result on the non-commuting graphs by answering a problem of Erdős. From that time on, noncommuting graphs have been studied extensively in the literature ${ }^{5,6}$.

The aim of this paper is to give a generalization of the non-commuting graph of a group $G$ with respect to a given normal subgroup $N$ of $G$. In the next section, we introduce the generalized noncommuting graph $G$ through a normal subgroup $N$ of $G$ denoted by $\Gamma_{N, G}$ and state some of the basic graph theoretical properties of this graph which are mostly new or a generalization of some results in Ref. 5 . We also give a connection between $\Gamma_{N, G}$ and the probability that the commutator of two arbitrary elements of $G$ belongs to the normal subgroup $N$ of $G$. We also state some conditions under which the graph is regular or strongly regular.

\section{GENERALIZED NON-COMMUTING GRAPH}

Definition 1 Let $G$ be a finite group and $N$ be a normal subgroup of $G$. The non-commuting graph of $G$ can be generalized using the subgroup $N$ is such a way that its vertices are $G \backslash Z^{N}(G)$ where $Z^{N}(G)=\{g \in G: g N \in Z(G / N)\}$ and two distinct vertices are adjacent when $[x, y] \notin N$. This graph is denoted by $\Gamma_{N, G}$.

Notice that if $N=1$ then the graph $\Gamma_{N, G}$ is the ordinary non-commuting graph of $G$. Clearly, $N \subseteq$ $Z^{N}(G)$ and so the elements of $N$ do not belong to the vertex set of $\Gamma_{N, G}$. One can easily see that the graph $\Gamma_{N, G}$ is null whenever $G$ is abelian or $N=G$. Hence, throughout this paper, it is always assumed that $G$ is a non-abelian group and $N$ is a proper normal subgroup of $G$.

We also note that if $[G: N]=k$ and $\left\{x_{1}, \ldots, x_{k}\right\}$ is a left transversal of $N$ in $G$ such that $\left[x_{i}, x_{j}\right] \in N$ for all $1 \leqslant i, j \leqslant k$, then $\Gamma_{N, G}$ is a null graph.

Example 1 Let $D_{12}=\langle a, b| a^{6}=b^{2}=1 ; b a b=$ $\left.a^{-1}\right\rangle$ be the dihedral group of order 12 and put $N=Z\left(D_{12}\right)=\left\{e, a^{3}\right\}$. Then $\Gamma_{D_{12, N}}$ is drawn as in Fig. 1.

Lemma 1 Let $G$ be a group and $N$ be a normal 


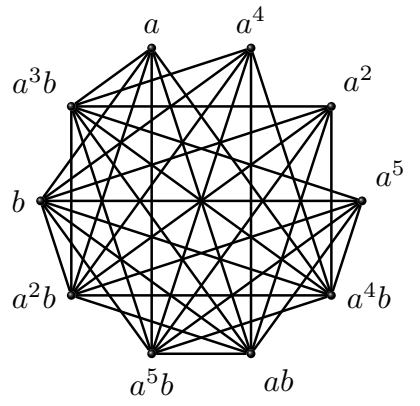

Fig. 1 Generalized non-commuting graph $\Gamma_{D_{12, N}}$.

subgroup of $G$. If $x, y \in G$, then $[x, y] \notin N \Leftrightarrow$ $\left[x n, y n^{\prime}\right] \notin N$ for all $n, n^{\prime} \in N$.

By the above lemma, it can be easily checked that the graph $\Gamma_{N, G}$ does not have any isolated vertex and is never an empty graph.

Definition 2 Let $G$ be a group and $N$ be a normal subgroup of $G$. Then for every $x \in G$,

$$
C_{G}^{N}(x)=\left\{g \in G: g N \in C_{G / N}(x N)\right\} .
$$

It is clear that $C_{G}^{N}(x)$ is a subgroup of $G$ and $\operatorname{deg}(x)=|G|-\left|C_{G}^{N}(x)\right|$ for all $x \in V\left(\Gamma_{N, G}\right)$.

Theorem 1 Let $G$ be a finite group and $N$ be a nontrivial subgroup of $G$. Then $\Gamma_{N, G}$ is not a complete graph.

Proof: Suppose on the contrary that $\Gamma_{N, G}$ is complete. Then

$$
\operatorname{deg}(h)=|G|-\left|C_{G}^{N}(h)\right|=|G|-\left|Z^{N}(G)\right|-1
$$

for all vertices $h$ of $\Gamma_{N, G}$. Then $\left|C_{G}^{N}(h)\right|=\left|Z^{N}(G)\right|+1$. On the other hand, $\left|Z^{N}(G)\right|$ divides $\left|C_{G}^{N}(h)\right|$. Thus $\left|Z^{N}(G)\right|=1$ which contradicts the choice of $N$.

Theorem $2 \operatorname{diam}\left(\Gamma_{N, G}\right)=2$ and $\operatorname{girth}\left(\Gamma_{N, G}\right)=3$.

Proof: Let $a, b$ be two non-adjacent vertices. Then there exist $x, y$ such that $[a, x] \notin N$ and $[b, y] \notin$ $N$. Now if $a$ is adjacent to $y$ or $b$ is adjacent to $x$, then $d(x, y)=2$. Suppose $[a, y] \in N$ and $[b, x] \in$ $N$. It is obvious that $x y$ is a vertex and therefore $x y$ is adjacent to both $x$ and $y$. Hence $\operatorname{diam}\left(\Gamma_{N, G}\right)=$ 2 .

Theorem 3 A lower bound for the minimum degree of vertices of $\Gamma_{N, G}$, which we denote by $\delta\left(\Gamma_{N, G}\right)$, satisfies $\delta\left(\Gamma_{N, G}\right) \geqslant 3|N|$.
Proof: Let $x$ be an arbitrary vertex of $\Gamma_{N, G}$. Since $\Gamma_{N, G}$ does not have any isolated vertex, there exists a vertex $y$ adjacent to $x$. Hence $x \sim n y$ for all $n \in$ $N$. Since $x y$ is a vertex of $\Gamma_{N, G}$ adjacent to $x$, we have $x \sim n x y$ for all $n \in N$. As $x y \neq y x, x \sim n y x$ for all $n \in N$, from which it follows that $\delta\left(\Gamma_{N, G}\right) \geqslant$ $3|N|$.

Proposition 1 Let $G$ be a non-abelian group and $N$ be a normal subgroup of $G$. Then $\Gamma_{N, G}$ is Hamiltonian.

Proof: One can easily see that $\operatorname{deg}(x) \geqslant\left|V\left(\Gamma_{N, G}\right)\right| / 2$ for all $x \in V\left(\Gamma_{N, G}\right)$. The proof is similar to that for Proposition 2.2 in Ref. 5.

An Eulerian tour is a walk which passes every edge exactly once. A graph is Eulerian if it contains an Eulerian tour. In the following propositions, we determine some conditions for $N$ and $G$ when the graph $\Gamma_{N, G}$ is Eulerian.

Proposition 2 If $|N|$ is even, then $\Gamma_{N, G}$ is Eulerian.

Proof: Let $V\left(\Gamma_{N, G}\right)=x_{1} N \cup \cdots \cup x_{r} N$. Let $x$ be any vertex of $\Gamma_{N, G}$ and $x_{i_{1}}, \ldots, x_{i_{t}}$ be those vertices among $x_{1}, \ldots, x_{r}$ adjacent to $x$. Hence $x \sim n x_{i_{j}}$ for all $n \in N$ and $1 \leqslant j \leqslant t$, which implies that $\operatorname{deg}(x)=t|N|$ is even. Hence by Theorem 4.1 in Ref. 7 the result follows.

Proposition 3 If $|G|$ is odd, then $\Gamma_{N, G}$ is Eulerian.

Proof: Let $x \in V\left(\Gamma_{N, G}\right)$ be an arbitrary vertex. We know that $\operatorname{deg}(x)=|G|-\left|C_{G}^{N}(x)\right|$. Since $C_{G}^{N}(x)$ is a subgroup of $G$, its order is odd and hence $\operatorname{deg}(x)$ is even. The result follows by Theorem 4.1 in Ref. 8.

In the following theorem, we give a lower bound for the chromatic number $\chi\left(\Gamma_{N, G}\right)$ of the graph $\Gamma_{N, G}$.

Theorem 4 Suppose $[G: N]=k+1$ and $G=x_{0} N \cup$ $x_{1} N \cup \cdots \cup x_{k} N$. Then

$$
\begin{aligned}
\chi\left(\Gamma_{N, G}\right) \geqslant 1+\max \{|A| & : A \subseteq\left\{x_{0}, \ldots, x_{k}\right\}, \\
& \left.x_{i}, x_{j} \in A,\left[x_{i}, x_{j}\right] \notin N,\right\} .
\end{aligned}
$$

Proof: Put $t_{1}=\left|\left\{x_{i}:\left[x_{i}, x_{1}\right] \notin N\right\}\right|$. Then it is clear that $\chi\left(\Gamma_{N, G}\right) \geqslant t_{1}+1$. Similarly, if $t_{j}=\mid\left\{x_{i}\right.$ : $\left.\left[x_{i}, x_{j}\right] \notin N\right\} \mid$, then we need at least $t_{j}+1$ colours in order to colour $\Gamma_{N, G}$ and again $\chi\left(\Gamma_{N, G}\right) \geqslant t_{j}+1$. Now, if $t=\max \left\{t_{j}: 1 \leqslant j \leqslant k\right\}$, then $\chi\left(\Gamma_{N, G}\right) \geqslant t+1$, as required.

We now may state some results for the dominating number of the graph $\Gamma_{N, G}$. A subset of the graph is a dominating set if every vertex which is not in the subset is adjacent to at least one member of the subset. The size of the minimum dominating set is called the dominating number. 
Theorem 5 Let $G$ be a non-abelian group and $N$ be a normal subgroup of $G$ such that $x N$ is a dominating set for $\Gamma_{N, G}$ for every vertex $x$ of $\Gamma_{N, G}$. Then $Z^{N}(G)=$ $N, x^{2}=1$, and $C_{G / N}=\langle x N\rangle$.

Proof: To prove $Z^{N}(G)=N$, assume on the contrary that $Z^{N}(G) \neq N$. Then there exists an element $a \in$ $Z^{N}(G)-N$ and so $[a, g] \in N$ for all $g \in G$. Since $x$ is a vertex, $x \notin Z^{N}(G)$ which implies that $[x, b] \notin N$ for some $b \in G$. Thus $[x a, b] \notin N$. Consequently, $x a$ is a vertex. We now have $[x a, x]=[a, x] \in N$ and so $x a$ is not adjacent to $x$ which is a contradiction. If $x^{2} \neq 1$, then $x^{-1} \neq x$ and again $x^{-1}$ is not adjacent to $x$ is a contradiction. The last part directly follows from the point that $x$ is a vertex in the dominating set and $x^{2}=1$.

One can easily see that a subset $S$ of $V\left(\Gamma_{N, G}\right)$ is a dominating set if and only if $C_{G}^{N}(S) \subseteq Z^{N}(G) \cup S$. Also, if $X$ is a generating set for $G$, then $X N \backslash Z^{N}(G) N$ is a dominating set for $\Gamma_{N, G}$.

A cut-set of $\Gamma_{N, G}$ is a set of edges of the graph which, if removed, disconnects the graph. The vertex connectivity of $\Gamma_{N, G}$, denoted by $\kappa\left(\Gamma_{N, G}\right)$, is the minimum size of all cut sets. In the following proposition we determine the vertex connectivity of $\Gamma_{N, G}$.

Proposition 4 Let $G$ be a non-abelian group and $S$ be a cut set of $\Gamma_{N, G}$. If $x, y \in V\left(\Gamma_{N, G}\right) \backslash S$ belong to distinct components of $\Gamma_{N, G} \backslash S$ then $S$ can be written as a union of double cosets of the subgroup $C_{G}^{N}(x) \cap C_{G}^{N}(y)$. Also, if $G$ is finite then $\kappa\left(\Gamma_{N, G}\right)=t\left|Z^{N}(G)\right|$, in which $t$ is an integer greater that 1 .

Proof: It is similar to the proof of Proposition 2.4 in Ref. 5. Put $H=C_{G}^{N}(x) \cap C_{G}^{N}(y)$. Since $x$ and $y$ are vertices, $x, y \notin Z^{N}(G)$ and consequently, $H \neq G$. We now prove that $S=\bigcup_{a \in G} \mathrm{HaH}$. Firstly, we show that for every $a \in G$, if $H a H \cap S \neq \varnothing$, then $H a H \subseteq S$. On the contrary, assume that $\mathrm{HaH} \nsubseteq S$. Then there exist elements $h_{1}, h_{2} \in H$ such that $h_{1} a h_{2} \notin S$. It can be easily seen that $x \sim h_{1} a h_{2} \sim y$ which is a contradiction. Hence $\bigcup_{a \in G} H a H \subseteq S$ and $S \subseteq$ $G=\bigcup_{a \in G} H a H$ which imply that $S=\bigcup_{a \in G} H a H$ whenever $H a H \cap S \neq \varnothing$. Secondly, assume that $k\left(\Gamma_{N, G}\right)=|S|$, where $S$ is a minimum cut set of $\Gamma_{N, G}$. Then $Z^{N}(G) \leqslant H$ allows as to write $S$ as the union of cosets of $Z^{N}(G)$. Thus there exists a positive integer $t$ such that $k\left(\Gamma_{N, G}\right)=t\left|Z^{N}(G)\right|$. We claim that $t>1$. If $t=1$ then $S=b Z^{N}(G)$ for some $b \notin Z^{N}(G)$. If $x_{1}$ and $y_{1}$ and two vertices belong to different connected components of $\Gamma_{N, G} \backslash S$ then $[x, y] \in N$. Since $\operatorname{diam}\left(\Gamma_{N, G}\right)=2$, we should have a path between $x_{1}$ and $y_{1}$ which is a contradiction. Hence $t>1$.

The commutativity degree $d(G)$ of a finite group $G$ is the probability that two randomly chosen elements of $G$ commute. We may extend it to the commutativity degree of $G$ with respect to a normal subgroup $N$ of $G$, denoted by $d^{N}(G)$ :

$$
d^{N}(G)=\frac{|\{(x, y) \in G \times G:[x, y] \in N\}|}{|G|^{2}} .
$$

It is obvious that if $N=1$, then $d(G)=d^{N}(G)$. Also, one can see that $d^{N}(G)=d(G / N)$. Utilizing the above definition, we may find a formula for the number of edges of $\Gamma_{N, G}$.

Lemma 2 The number of edges of $\Gamma_{N, G}$ is $\frac{1}{2}|G|^{2}(1-$ $\left.d^{N}(G)\right)$.

Proof: Let $A=\{(x, y) \in G \times G \mid[x, y] \in N\}$ and $B=$ $\{(x, y) \in G \times G \mid[x, y] \notin N\}$. It is easy to see that $d^{N}(G)=|A| /|G|^{2}$. On the other hand,

$$
\begin{aligned}
|G|^{2}= & |\{(x, y) \in G \times G:[x, y] \in N\}| \\
& +|\{(x, y) \in G \times G:[x, y] \notin N\}| \\
= & |A|+|B| .
\end{aligned}
$$

Also, as $|B|=2\left|E\left(\Gamma_{N, G}\right)\right|$, we have

$$
2\left|E\left(\Gamma_{N, G}\right)\right|=|B|=|G|^{2}-|A|^{2}=|G|^{2}\left(1-d^{N}(G)\right)
$$

and the result follows.

Using the above lemma, we obtain the following inequalities.

Proposition 5 Let $G$ be a finite group and $N$ be a normal subgroup of $G$. Then

(i) $d^{N}(G) \geqslant 2\left|Z^{N}(G)\right| /|G|+1 /|G|-\left|Z^{N}(G)\right|^{2} /|G|^{2}-$ $\left|Z^{N}(G)\right| /|G|^{2}$

(ii) $\left|E\left(\Gamma_{N, G}\right)\right| \geqslant \frac{3}{16}|G|^{2}$, if $G / N$ is not abelian.

Proof: They follow from Lemma 2 and an upper bound $\frac{5}{8}$ for $d(G)$ by a result in Ref. 9 .

A graph is $k$-regular if every vertex has degree $k$. Also, a $k$-regular graph with $n$ vertices is called strongly regular with parameter $(n, k, r, s)$ if it is neither empty nor complete such that any two adjacent vertices are adjacent to $r$ common vertices and any two non-adjacent vertices are adjacent to $s$ common vertices.

Theorem 6 If $\Gamma_{N, G}$ is a k-regular graph, then $|N|$ divides $k$.

Proof: Let $x \in V\left(\Gamma_{N, G}\right)$ and $y$ be a vertex adjacent to $x$. Then $x \sim y n$ for all $n \in N$ so that $|N|$ divides $\operatorname{deg}(x)$. Since $\operatorname{deg}(x)=k$, it follows that $|N|$ divides $k$, as required. 
Theorem 7 If $\Gamma_{N, G}$ is strongly regular with parameters $(n, k, r, s)$, then $s=k$.

Proof: Let $x \in V\left(\Gamma_{N, G}\right)$ and $z \in Z^{N}(G) \backslash N$. Clearly, $x z \in V\left(\Gamma_{N, G}\right)$. Since $x$ and $x z$ are not adjacent, $s=$ $\left|G \backslash\left(C_{G}^{N}(x) \cup C_{G}^{N}(x z)\right)\right|$. Since $C_{G}^{N}(x)=C_{G}^{N}(x z)$ and $\Gamma_{N, G}$ is $k$-regular, the result follows.

To prove the next theorem, we need the following lemma concerning AC-groups. A group is an ACgroup if its nontrivial centralizers are abelian.

Lemma 3 The following statements are equivalent:

(i) $G$ is an AC-group;

(ii) if $x, y \in G \backslash Z(G)$ such that $[x, y]=1$, then $C_{G}(x)=C_{G}(y)$

(iii) if $x, y, z \in G \backslash Z(G)$ such that $[x, y]=[y, z]=1$, then $[x, z]=1$;

(iv) if $A$ and $B$ are subgroups of $G$ such that $Z(G) \subset$ $C_{G}(A) \subseteq C_{G}(B) \subset G$, then $C_{G}(A)=C_{G}(B)$.

Proof: See Lemma 3.2 in Ref. 10.

Corollary 1 If $\Gamma_{N, G}$ is a strongly regular graph with parameters $(n, k, r, s)$, then $G / N$ is an AC-group.

Proof: Let $x, y$ be two non-adjacent vertices. Then $C_{G / N}(x N)=C_{G / N}(y N)$ since $\operatorname{deg}(x)=\left|G \backslash C_{G}^{N}(x)\right|$ and $\operatorname{deg}(y)=\left|G \backslash C_{G}^{N}(y)\right|$ and hence $\left|C_{G}^{N}(x)\right|=$ $\left|C_{G}^{N}(y)\right|$. As $k=|G|-\left|C_{G}^{N}(x)\right|=|N(x) \cap N(y)|=$ $\left|G \backslash C_{G}^{N}(x) \cup C_{G}^{N}(y)\right|$, we conclude that $\left|C_{G}^{N}(x)\right|=$ $\left|C_{G}^{N}(x) \cup C_{G}^{N}(y)\right|$. Hence $C_{G}^{N}(y) \subseteq C_{G}^{N}(x)$ and consequently $C_{G}^{N}(x)=C_{G}^{N}(y)$. Thus $C_{G}^{N}(x) / N=$ $C_{G}^{N}(y) / N$. Hence $C_{G / N}(N)=C_{G / N}(y N)$ and, by Lemma $3, G / N$ is an AC-group. Let $x, y$ be two non-adjacent vertices. Then $[x, y] \in N$. Since $\operatorname{deg}(x)=\left|G \backslash C_{G}^{N}(x)\right|=\left|G \backslash C_{G}^{N}(y)\right|$ and $\Gamma_{N, G}$ is regular, we have $\left|C_{G}^{N}(x)\right|=\left|C_{G}^{N}(y)\right|$. Assume that $t \in G \backslash\left(C_{G}^{N}(x) \cup C_{G}^{N}(y)\right)$. Then $t$ is adjacent to $x$ and $y$. The number of common neighbours of $x$ and $y$ are equal and is $k$, so we should have $k=$ $\left|G \backslash\left(C_{G}^{N}(x) \cup C_{G}^{N}(y)\right)\right|=\left|G \backslash C_{G}^{N}(x)\right|$. Consequently, $\left|C_{G}^{N}(x)\right|=\left|C_{G}^{N}(x) \cup C_{G}^{N}(y)\right|$ and so $C_{G}^{N}(x)=C_{G}^{N}(y)$. Hence $C_{G \backslash N}^{N^{G}}(x N)=C_{G \backslash N}^{N}(y N)$ and by Lemma 3, $G / N$ is an AC-group as required.

Theorem 8 If $\Gamma_{N, G}$ is a strongly regular graph with parameters $(n, k, r, s)$ then $r=2 k-n$.

Proof: Let $x, y$ be two adjacent vertices. Then $[x, y] \notin N$. Hence, $C_{G}^{N}(x) \cap C_{G}^{N}(y)=Z^{N}(G)$, by Corollary 1 and a consequence of Lemmas 4.2 and
4.3 in Ref. 5. We now have

$$
\begin{aligned}
r & =|N(x) \cap N(y)| \\
& =\left|G \backslash C_{G}^{N}(x) \cup C_{G}^{N}(y)\right| \\
& =|G|-\left|C_{G}^{N}(x)\right|-\left|C_{G}^{N}(y)\right|+\left|C_{G}^{N}(x) \cap C_{G}^{N}(y)\right| \\
& =|G|-\left|C_{G}^{N}(x)\right|+|G|-\left|C_{G}^{N}(y)\right|+\left|Z^{N}(G)\right|-|G| \\
& =2 k-n,
\end{aligned}
$$

where $N(x)$ is the set of neighbours of $x$.

In the following two propositions, we give a link between this graph and the nilpotency property in group theory.

Proposition 6 Suppose $G=x_{1} N \cup x_{2} N \cup \ldots \cup x_{k} N$ such that $x_{i}$ is a vertex in $\Gamma_{N, G}$, for each $2 \leqslant i \leqslant k$. Then $G$ is not nilpotent.

Proof: Since all $x_{i}$ for $2 \leqslant i \leqslant k$ are vertices, $Z^{N}(G)=N$ and therefore $|Z(G / N)|=1$. Hence $G$ cannot be nilpotent.

Proposition 7 Let $N$ be a normal subgroup of $G$ and $G / N$ be non-abelian. If $\Gamma_{N, G}$ is regular, then $G / N$ is a nilpotent group.

Proof: For every vertex $x$ in $\Gamma_{N, G}$ we have $\operatorname{deg}(x)=$ $|G|-\left|C_{G}^{N}(x)\right|$. Moreover, if $y \notin Z^{N}(G)$, then $\operatorname{deg}(x)=\operatorname{deg}(y)$ which implies that $\left|C_{G}^{N}(x)\right|=$ $\left|C_{G}^{N}(y)\right|$. On the other hand, if $z N \in Z(G / N)$, then $C_{G / N}(z N)=G / N$ and consequently, the size of all conjugacy classes of $G / N$ has only two values: 1 and a positive integer $n \neq 1$. Hence $G / N$ is a nilpotent by a theorem of Ito $^{11}$.

Recall that a graph is planar if it can be drawn in the plane such that edges intersect only at vertices. The planarity of $\Gamma_{N, G}$ is investigated in Ref. 5 when $N=1$ is the trivial subgroup. In the following theorem, we show that $\Gamma_{N, G}$ is not planar when $N \neq 1$.

Theorem 9 The graph $\Gamma_{N, G}$ is never planar for every nontrivial normal subgroup $N$ of $G$.

Proof: Let $N \neq 1$ and $\Gamma_{N, G}$ be planar. Then $\delta\left(\Gamma_{N, G}\right) \leqslant$ 5 (see Corollary 3.5.9 in Ref. 8). On the other hand, $\delta\left(\Gamma_{N, G}\right) \geqslant 3|N|$ by Theorem 3. Hence $3|N| \leqslant 5$, which is a contradiction.

A graph is called 1-planar if it can be drawn in the plane in such a way that each edge is crossed by no more than one other edge. Note that graphs $K_{7}$, $K_{3,7}$ and $K_{4,5}$ are not 1-planar in Ref. 12.

Lemma 4 There is a vertex in $\Gamma_{N, G}$ whose order in $G$ is greater than 2 . 


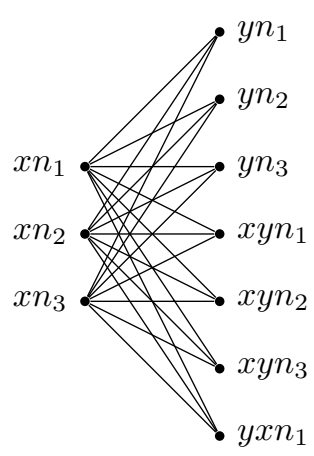

Fig. 2 Induced subgraph of $\Gamma_{N, G}$ isomorphic to $K_{3,7}$.
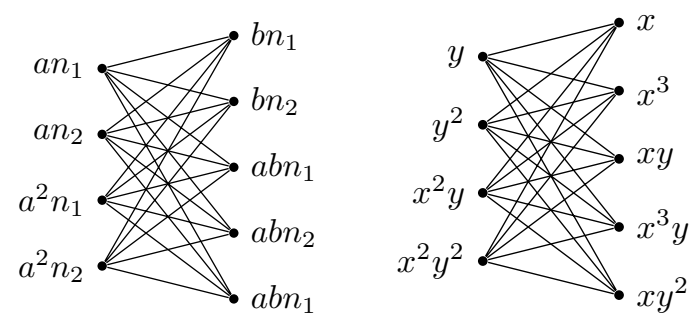

Fig. 3 Induced subgraphs of $\Gamma_{N, G}$ isomorphic to $K_{4,5}$.

Proof: Suppose on the contrary that $x^{2}=1$ for all $x \in V\left(\Gamma_{N, G}\right)$. Let $x$ and $y$ be two adjacent vertices. Then $[x, y] \notin N$ and hence $1=(x y)^{2} \notin N$ which is a contradiction.

Theorem 10 The graph $\Gamma_{N, G}$ is 1-planar if and only if it is planar and hence $G \cong S_{3}, D_{8}, Q_{8}$, and $N=1$.

Proof: If $|N| \geqslant 4$ then $\Gamma_{N, G}$ has a subgraph isomorphic to $K_{4,5}$ and hence it is not a 1-planar graph. Also, if $N=\left\{n_{1}, n_{2}, n_{3}\right\}$ and $x, y$ are two adjacent vertices, then $\Gamma_{N, G}$ has a subgraph isomorphic to $K_{3,7}$ as drawn in Fig. 2. Hence $\Gamma_{N, G}$ is not 1-planar.

If $|N|=2$ then, by Lemma 4 , the graph $\Gamma_{N, G}$ has a vertex $a$ satisfying $|a|>2$. Let $b$ be a vertex adjacent to $a$. If $N=\left\{n_{1}, n_{2}\right\}$, then $\Gamma_{N, G}$ has a subgraph isomorphic to $K_{4,5}$ as shown in the left of Fig. 3. Hence $\Gamma_{N, G}$ is not 1-planar.

Finally, if $|N|=1$, then we show that $\Gamma_{N, G}$ is not a 1-planar graph. Suppose on the contrary that $\Gamma_{N, G}$ is 1-planar. Since the complete graph with 7 vertices is not a 1-planar graph, we must have $w\left(\Gamma_{N, G}\right) \leqslant 6$. As in the proof of Proposition 2.3 in Ref. 5, one can show that $|G| \leqslant 14$. Hence we only need to consider non-abelian groups of order less than or equal to 14, i.e., $S_{3}, D_{8}, Q_{8}, D_{10}, A_{4}, D_{12}, T=\left\langle x, y: x^{4}=y^{3}=\right.$ $\left.1, y^{x}=y^{-1}\right\rangle$ and $D_{14}$. If $G \cong S_{3}, D_{8}$ or $Q_{8}$, then $\Gamma_{N, G}$ is planar so that it is 1-planar. Now, if $G \cong D_{10}, A_{4}$ or $D_{12}$, then $\Gamma_{N, G}$ has a subgraph isomorphic to $K_{4,5}$.
Hence it is not a 1-planar graph. Also, if $G \cong T$, then $\Gamma_{N, G}$ has a subgraph isomorphic to $K_{4,5}$ as drawn in the right of Fig. 3. Hence it is not 1-planar.

Finally, if $G \cong D_{14}$, then $\Gamma_{N, G}$ has a subgraph isomorphic to $K_{7}$ so that it is not a 1-planar graph.

A graph is called outer planar if it can be drawn in the plane without crossing edges in such a way that all vertices belong to the unbounded face of the drawing.

Theorem 11 The graph $\Gamma_{N, G}$ is never an outer-planar graph.

Proof: If $|N| \geqslant 2$, then clearly $\Gamma_{N, G}$ is not planar by Theorem 9 and consequently is not outer planar. Thus we may assume that $N=1$ and that $\Gamma_{N, G}$ is outer planar. Hence $w\left(\Gamma_{N, G}\right) \leqslant 3$. As in the proof of Proposition 2.3 in Ref. 5, one can show that $|G| \leqslant$ 8. The only non-abelian groups of order less or equal to 8 are $S_{3}, D_{8}$ and $Q_{8}$ for which the graph $\Gamma_{N, G}$ has a subgraph isomorphic to $K_{2,3}$, which is a contradiction. Hence $\Gamma_{N, G}$ is not an outer planar graph.

\section{REFERENCES}

1. Williams JS (1981) Prime graph components of finite groups. J Algebra 69, 487-513.

2. Abdollahi A, Mohammadi Hassanabadi A (2007) Non-cyclic graph of a group. Comm Algebra 35, 2057-81.

3. Erfanian A, Tolue B (2012) Conjugate graphs of finite groups. Discrete Math Algorithm Appl 4, 1250035.

4. Neumann BH (1976) A problem of Paul Erdős on groups. $J$ Aust Math Soc Ser A 21, 467-72.

5. Abdollahi A, Akbari S, Maimani HR (2006) Noncommuting graph of a group. J Algebra 298, 468-92.

6. Solomon RM, Woldar AJ (2013) Simple groups are characterized by their non-commuting graphs. $J$ Group Theor 16, 793-824.

7. Tolue B, Erfanian A (2013) Relative non commuting graph of a finite group. J Algebra Appl 12, 1250157.

8. Bondy JA, Murty JSR (1997) Graph Theory with Applications, Elsevier.

9. Gustafson WH (1973) What is the probability that two group elements commute? Amer Math Mon 80, 1031-304.

10. Rocke DM (1975) p-groups with abelian centralizers. Proc Lond Math Soc 30, 55-75.

11. Ito N (1953) On finite groups with given conjugate types I. Nagoya Math J 6, 17-28.

12. Czap J, Hudák D (2012) 1-planarity of complete multipartite graphs. Discrete Appl Math 160, 505-12. 